\title{
A CLASS OF MAXIMAL IDEALS IN THE LATTICE OF TOPOLOGIES
}

\author{
W. J. THRON AND R. A. VALENT
}

\begin{abstract}
In this article we investigate maximal ideals in the lattice of all topologies on a fixed set. Padmanabhan and Rao were the first to study maximal ideals in this lattice. They characterized the principal maximal ideals. Their main result was incorrect but their approach proved helpful to us. We exhibit a class of nonprincipal maximal ideals and also give an example to show that the class in question is not exhaustive.
\end{abstract}

1. Introduction. During the 1940's E. Hewitt [2] and M. Katětov [3, 4] investigated topologies having no disjoint dense sets. Each found that many of the classical topologies (e.g. $T_{2}$, dense-in-itself, and bicompact) do not belong to this class; furthermore, they demonstrated that elements of the lattice of topologies which are maximal with respect to certain topological properties $P$ do not belong to this class. Among the properties they considered, Hewitt chose $P$ to be $T_{0}$ and dense-in-itself, whereas Katětov took $P$ as $T_{2}$ together with the property of having a designated set of isolated points. Most of the present knowledge about such $P$-maximal topologies may be found in the three articles cited.

In this paper we investigate maximal ideals in the lattice of topologies in the context of these maximal topologies. A. R. Padmanabhan and B. V. Rao [5] were the first to study maximal ideals in the lattice of topologies. Although their main result is incorrect [6], they did characterize the principal maximal ideals, and their approach using set ultrafilters has proved useful to us. The maximal ideals which we introduce here have a simple form akin to that of principal maximal ideals, and in this sense may be viewed as a natural extension of the class of principal maximal ideals. To our knowledge, this is the first time any nonprincipal maximal ideals have been displayed for the lattice of topologies, although the cardinality of the family of all maximal ideals is known [7]. In addition, we provide an example which shows that the class of all maximal ideals studied here is not exhaustive.

2. Maximal ideals and nearly discrete topologies. As usual, $\Sigma$ denotes the lattice of all topologies on a fixed set $X, \leqslant$ denotes the order relation of $\Sigma$, and $\vee$ denotes the supremum operation of $\Sigma$. Given $A \subseteq X$ and $\mathcal{T} \in \Sigma, \mathcal{T}(A)$ is the smallest topology on $X$ containing $\mathcal{T} \cup\{A\}$.

Received by the editors November 4, 1981 and, in revised form. April 26, 1982.

1980 Mathematics Subject Classification. Primary 54A10; Secondary 06B10, 06B30.

(c1983 American Mathematical Society 0002-9939/82/0000-0412/\$02.25 
Since $\Sigma$ has a largest element (the discrete topology $\mathcal{P}(X)$ ), Zorn's Lemma guarantees the existence of maximal ideals of $\Sigma$. O. Fröhlich [1] proved that $\Sigma$ is antiatomic, and therefore $\Sigma$ has maximal ideals which are principal. Fröhlich's characterization of the antiatoms is very useful in examining the structure of principal maximal ideals of $\Sigma$ : antiatoms of $\Sigma$ are topologies of the form $\mathcal{T}(a, \mathcal{Q})=$ $\mathscr{P}(X-\{a\}) \cup \mathcal{Q}$, where $a \in X$ and $\mathscr{Q}$ is an ultrafilter on $X$ distinct from the principal ultrafilter $\mathscr{F}_{a}=\{A \subseteq X \mid a \in A\}$. It is clear that $\mathscr{G}=\mathscr{P}(X-\{a\})$ is an ideal on $X$, and $\mathcal{J}=\mathscr{Q} \cup\{\varnothing\}$ is a topology on $X$. Therefore the principal maximal ideal [ $\mathcal{T}(a, \mathcal{Q})]$ may be written as $\{\mathcal{S} \in \Sigma \mid \mathcal{S} \leqslant \mathcal{T} \vee(\mathscr{P}(A) \cup\{X\})$ for some $A \in \mathcal{G}\}$. Note that $\mathscr{P}(A) \cup\{X\}$ is a topology on $X$, and $\mathcal{T} \vee(\mathscr{P}(A) \cup\{X\})$ is the smallest topology on $X$ containing $\mathcal{T}$ and the isolated points $x$, where $x \in A$.

The analysis of principal maximal ideals given by Fröhlich's characterization of antiatoms motivates the following definition:

Definition 1. For any ideal $\mathcal{G}$ on $X$ and any $\mathcal{T} \in \Sigma, \mathfrak{A}(\mathcal{G}, \mathcal{T})$ is the ideal $\{\delta \in \Sigma \mid \delta \leqslant \mathcal{T} \vee(\mathscr{P}(A) \cup\{X\})$ for some $A \in \mathcal{G}\}$. Any such ideal of $\Sigma$ is said to be principal modulo a set ideal.

Our main purpose in this paper is to determine when $\mathfrak{A}(\mathcal{G}, \mathcal{T})$ is maximal. Again taking a cue from the principal case, it is reasonable to expect that $\mathcal{T}$ be large in some sense. The precise meaning of this is explained in Definition 2.

Given topologies $R, \mathcal{S} \in \Sigma$, recall that $\mathcal{S}$ is an expansion of $R$ if $R \leqslant \mathcal{S}$ [2]. Merging Hewitt's definition of maximal topology [2] with Katětov's definition of nearly discrete topology [3], we obtain the class of topologies pertinent to our study. We use Katětov's terminology.

Definition 2. A $T_{0}$-topology $\mathcal{T}$ on $X$ is nearly discrete if every proper expansion of $T$ has at least one isolated point which is not isolated in $\mathcal{T}$.

The discrete topology and the antiatoms of $\Sigma$ are perhaps the simplest and best known of the nearly discrete topologies. By Zorn's Lemma, any $T_{0}$-topology $\Re \in \Sigma$ may be expanded to a nearly discrete topology $\mathcal{T} \in \Sigma$ having the same set of isolated points. An example of a $T_{1}$, nearly discrete topology having no isolated points is $\mathscr{U} \cup\{\varnothing\}$, where $\mathcal{Q}$ is a nonprincipal ultrafilter on $X$. There are examples of nearly discrete $T_{2}$-topologies devoid of isolated points, and it is an open question whether such $T_{3}$-topologies exist [4].

We shall have occasion to use the following strong property of nearly discrete topologies. This was proved by Katětov for the Hausdorff case [3].

LEMMA 1. Let $\mathcal{T}$ be a nearly discrete topology on $X$ and $A \subseteq X$. If $x$ is a limit point of $A$ in $\mathcal{T}$, then $A \cup\{x\}$ is a neighborhood of $x$ in $\mathcal{T}$.

Proof. As usual, $A^{\prime}$ denotes the set of limit points of $A$ in $\widetilde{T}$, and $\bar{A}$ denotes the closure of $A$ in $\mathcal{T}$. We first show that $A^{\prime} \cap \overline{\sim \bar{A}}=\varnothing$. Let $a \in \overline{\sim \bar{A}}$; then $\sim \bar{A} \cup\{a\}$ is open in $\mathcal{T}$ by the nearly discreteness of $\mathcal{T}$ and the fact that $\sim \bar{A}$ is open in $\mathcal{T}$. Since $\varnothing=(A-\{a\}) \cap(\sim \bar{A} \cup\{a\}), a \notin A^{\prime}$. Therefore $A^{\prime} \cap \overline{\sim \bar{A}}=\varnothing$.

Now we shall prove the lemma. Let $x \in A^{\prime}$. By the preceding fact, $x \in \sim \overline{\sim \bar{A}}$. Since $A \cup\{x\} \cup \sim \bar{A}$ is dense in $\mathcal{T}$, it is also open. Hence $\sim \bar{A} \cap(A \cup\{x\} \cup$ $\sim \bar{A}) \subseteq A \cup\{x\}$ is a neighborhood of $x$ in $\mathscr{T}$ and this completes the proof. 
We remark that the property given in Lemma 1 characterizes nearly discreteness in $T_{0}$-topologies, though we do not use this here. Furthermore, the $T_{0}$ requirement in Definition 2 is very important (cf. Example 3).

We now present our basic result concerning the role of nearly discrete topologies in maximal ideals of $\Sigma$.

THEOREM 1. Let 9 be a maximal ideal on $X$ and let $\mathcal{T} \in \Sigma$ be a nearly discrete topology such that the set $I(T)$ of isolated points of $\mathscr{T}$ satisfies $I(\mathcal{T}) \in \mathcal{G}$. Then $\mathcal{H}(\mathcal{G}, \mathcal{T})$ is a maximal ideal of $\Sigma$.

Proof. Since $I(\tilde{T}) \in \mathscr{G}$ and $\mathscr{G}$ is a proper ideal on $X, \mathscr{A}(\mathcal{G}, T)$ is a proper ideal of $\Sigma$. For any $\S \in \Sigma-\mathfrak{A}(\Psi, \widetilde{\mathcal{J}})$, we will show that $\mathscr{U}(\mathcal{G}, \mathcal{J}) \cup\{\delta\}$ is contained in no proper ideal of $\Sigma$ : Let $B=\left\{x \in X \mid x \notin{\overline{N_{x}-\{x\}}}^{\text {i. }}\right.$ for some neighborhood $N_{x}$ of $X$ in $\$$. By Lemma $1, \mathscr{Q P}(B) \subseteq \S \vee \mathcal{T}$. Furthermore, $B \neq \varnothing, B \notin \mathcal{G}$, otherwise $\S$ would be in $\mathscr{A}(\mathcal{G}, \mathcal{T})$. Hence $\sim B \in \mathcal{G}$ and $\mathcal{T} \vee(\mathcal{O P}(\sim B) \cup\{X\}) \in \mathscr{U}(\mathcal{Y}, \mathcal{T})$, and $\mathscr{P}(X)=\tilde{T} \vee(\mathscr{P}(\sim B) \cup\{X\}) \vee \mathcal{S}$. Therefore $\mathscr{A}(\mathscr{G}, \tilde{\mathcal{T}})$ is a maximal ideal of $\Sigma$, and this completes this proof.

EXAMPLE 1. Let $\mathscr{Q}, \mathscr{Q}$ be distinct, nonprincipal ultrafilters on $X$; set $\Psi=\mathcal{P}(X)-\vartheta_{1}$ and $\tilde{T}=\mathscr{Q} \cup \cup\{\varnothing\}$. Then $\mathcal{U}(9, \widetilde{T})$ is a maximal ideal of $\Sigma$.

Proof. This follows immediately from Theorem 1.

Example 1 is one of the simplest instances of a nonprincipal maximal ideal, but it is important in other respects. It is a maximal ideal of $\Sigma$ having $T_{1}$ elements but no $T_{2}$ elements. Also, it answers the question posed by Padmanabhan and Rao in [6]. The problem may be stated as follows: Let $\$$ be a nonprincipal maximal ideal on $X$ and define $\mathcal{H}(\dot{y})=\{\delta \in \Sigma \mid \delta \leqslant(\stackrel{\mathcal{P}}{ }(A) \cup\{X\}) \vee\{\varnothing, \sim A, X\}$ for some $A \in \dot{\xi}\}$. Does every nonprincipal maximal ideal of $\Sigma$ contain some $\mathscr{H}(\mathcal{f})$ ? The answer is no. Otherwise, it would be the case that every nonprincipal maximal ideal $\mathscr{A}$ of $\Sigma$ has the property that $\{\varnothing, A, X\} \in \mathfrak{U}$ for all $A \subseteq X$. However, in Example 1 above, $\{\varnothing, A, X\} \notin \mathscr{U}(\Psi, \mathcal{J})$ for any $A \in$ Ql $\cap(\mathcal{Q P}(X)-\vartheta$

Our next goal is to characterize the class of maximal ideals which are principal modulo some set ideal. For this, it is helpful to know the following seemingly unrelated fact.

THEOREM 2. Every maximal ideal $\mathfrak{H}$ is $\Sigma$ has a $T_{0}$ element.

Proof. If $\mathscr{H}$ has a $T_{1}$ element, then it has a $T_{0}$ element. Suppose $\mathfrak{A}$ has no $T_{1}$ element. Then the minimum $T_{1}$-topology $\mathcal{e}$ on $X$ is not in $\mathscr{H}$. So there exists $: T \in \mathscr{H}$ such that $T \vee E=S P(X)$. It follows that $T$ has a base of finite sets.

Let $\equiv$ be the equivalence relation on $X$ given by $x \equiv y$ if and only if the neighborhood filters $\Re(x, \bar{T})=\Re(y, T)$. Since each $x \in X$ has a finite neighborhood in $\bar{\tau}, x / \equiv$ is finite. For each $x / \equiv$ such that $|x| \equiv \mid \geqslant 2$, fix a listing $x_{1}, \ldots, x_{n}$ of $x / \equiv$. Let $\tilde{T}^{*}$ be the smallest topology on $X$ containing $v^{\top} \cup$ $\left(\cup\left\{\left\{x_{1}\right\},\left\{x_{1}, x_{2}\right\}, \ldots,\left\{x_{1}, \ldots, x_{n}\right\}|| x / \equiv \mid \geqslant 2\right\}\right)$. Then $T^{*}$ is a $T_{0}$-topology on $X$. If $: T^{*} \in \mathscr{U}$, the assertion is proved.

If $\tau^{*} \notin \mathscr{U}$, there exists $S \in \mathscr{U}$ such that $T \leqslant S$ and $S \vee T *=\varphi(X)$. We claim that $\tilde{S}$ is $T_{0}$. Fix $x / \equiv$ with $|x / \equiv| \geqslant 2$. Since $S \vee T^{*}=? P(X)$, for each $i \in\{2, \ldots, n\}$ 
there exists $N_{x_{i}} \in S$ such that $\left\{x_{1}, \ldots, x_{i-1}\right\} \cap N_{x_{i}}=\varnothing$. Therefore, given $x_{j}, x_{k} \in$ $\left\{x_{1}, \ldots, x_{n}\right\}$ with $j<k$, there exists $N_{x_{k}} \in \mathfrak{s}$ such that $x_{j} \notin N_{x_{k}}$. Since $\tilde{g} \leqslant \xi$, it follows that for each $x_{i} \in\left\{x_{1}, \ldots, x_{n}\right\}$ and each $y \in X-\left\{x_{1}, \ldots, x_{n}\right\}$, there exist $N_{x,}, N_{y} \in S$ such that $\left\{x_{i}, y\right\} \nsubseteq N_{x,} \cap N_{y}$. Therefore $\delta \in \mathfrak{A}$ is $T_{0}$, and this completes the proof.

Our final theorem characterizes the maximal ideals which are principal modulo some set ideal. It guarantees that the combination of maximal set ideals with nearly discrete topologies as in Theorem 1 produces all maximal ideals which are principal modulo a set ideal.

THEOREM 3. Let $\mathfrak{A}$ be a maximal ideal of $\Sigma$. Then $\mathfrak{A}$ is principal modulo a set ideal if and only if $\mathfrak{H}=\mathfrak{H}(4, T)$ for some maximal ideal $\$$ on $X$ and some nearly discrete topology $\because$ on $X$ such that $I(T) \in 9$.

Proof. If $\mathfrak{A}=\mathfrak{A}(9, \pi)$, then $\mathfrak{A}$ is certainly principal modulo a set ideal by Definition 1. On the other hand, suppose $\mathscr{A}$ is maximal and principal modulo some set ideal. Let $\mathscr{Y}(\mathfrak{H})=\{A \subseteq X \mid \mathcal{P}(A) \cup\{X\} \in \mathfrak{H}\}$. Since $\mathfrak{A}$ is maximal, $\mathscr{Y}(\mathfrak{H})$ is a maximal ideal on $X$. By Definition $1, \mathfrak{A}=\mathfrak{A}(9(\mathfrak{A}), \widehat{s})$ for some $\delta \in \Sigma$. By Theorem 2 we may assume $S$ is $T_{0}$. By Zorn's Lemma $\$$ may be expanded to a topology $\tau^{*}$ on $X$ which is nearly discrete and has the same set of isolated points as $\$$. Then $\mathfrak{H}=\mathfrak{H}(\Psi(\mathfrak{H}), \bar{S}) \subseteq \mathfrak{H}\left(\Psi(\mathfrak{H}), \widetilde{T}^{*}\right)$, and by Theorem 1 this latter ideal is proper. Hence $\mathfrak{H}=\mathfrak{A}\left(\Psi(\mathfrak{U}), \widetilde{T}^{*}\right)$ and the proof is complete.

EXAMPLE 2. Not every maximal ideal of $\Sigma$ is principal modulo a set ideal.

Proof. We shall exhibit this ideal by defining a family $\Delta$ of topologies $\widetilde{T}_{C} \in \Sigma$ such that $\Delta$ can be extended to a maximal ideal $\mathfrak{A}$ of $\Sigma$. Then we shall prove $\mathfrak{A}$ is not principal modulo a set ideal. Let $X=\mathbf{N}=\{1,2,3, \ldots\}$, and let $\left\{a_{n}\right\}_{n=1}^{\infty}$ be any sequence from $\mathbf{N}$ such that (1) $a_{n}>1$, (2) $a_{n+1}>a_{n}$ for all $n \in \mathbf{N}$. and (3) for each $m \in \mathbf{N}$ there exists $n \in \mathbf{N}$ such that $a_{n+1}-a_{n}>m$. Define $A_{1}=\left\{1, \ldots, a_{1}\right\}$, and $A_{n+1}=\left\{a_{n}+1, \ldots, a_{n+1}\right\}$ for each $n \in \mathbf{N}$, and $\mathcal{G}^{\prime}=\left\{A_{n}-\left\{a_{n}\right\} \mid n \in \mathbf{N}\right\}$. For each choice set $C$ on $\mathcal{U}^{\prime}$ (i.e. each $C \subseteq \cup \mathcal{Q}^{\prime}$ such that $\left|C \cap\left(A_{n}-\left\{a_{n}\right\}\right)\right|=1$ for all $n \in \mathbf{N})$, define a topology $\widetilde{T}_{C}$ on $X$ as that topology having base $\{\varnothing\} \cup\left\{A_{n}-C \mid n\right.$ $\in \mathbf{N}\} \cup\left\{\{m\} \mid m \neq a_{n}\right.$ for any $\left.n \in \mathbf{N}\right\}$. By property (3) of $\left\{a_{n}\right\}_{n=1}^{\infty}$, no finite supremum $V_{1=1}^{k}, T_{C}$ is discrete. Hence $\Delta=\left\{\widetilde{T}_{C} \mid C\right.$ is a choice set on $\left.Q\right\}$ is contained in a proper ideal $\mathfrak{A}_{0}$ of $\Sigma$. By Zorn's Lemma, extend $\mathfrak{A}_{0}$ to a maximal ideal $\mathfrak{A}$ of $\Sigma$. We claim that $\mathfrak{A} \neq \mathfrak{A}(9, \widetilde{T})$ for any ideal $\mathscr{G}$ and any topology $\mathscr{T}$ on $X$. Otherwise, we may assume $T_{C} \leqslant T$ for some choice set $C$ on $Q$. Further, by Theorem 3 we may assume $\widetilde{T}$ is nearly discrete. Since $\widetilde{T}$ is nearly discrete and each $x \in X$ has a finite neighborhood in $\widetilde{T}$, it is true that each $x \in X$ has a neighborhood $N_{x}$ with $\left|N_{x}\right| \leqslant 2$. Define a choice set $B$ on $Q$ as follows: for each $n \in \mathbf{N}$ let

$$
b_{n} \in \begin{cases}N_{a_{n}}-\left\{a_{n}\right\} & \text { if } N_{a_{n}}-\left\{a_{n}\right\} \neq \varnothing, \\ A_{n}-\left\{a_{n}\right\} & \text { otherwise. }\end{cases}
$$

Letting $B=\left\{b_{n} \mid n \in \mathbf{N}\right\}$, it follows that $T \vee \mathcal{T}_{B}=\mathcal{P}(X)$, contradiction. Hence $\mathfrak{A} \neq \mathfrak{U}(9, \widetilde{T})$, and the verification is complete. 
We note that this example may be modified for any infinite $X$, and that we have produced similar examples with $T_{1}$ elements.

We now discuss the relevance of the $T_{0}$ requirement in the definition of nearly discrete topology: if the topology under consideration is not required to be $T_{0}$. neither Lemma 1 nor Theorem 1 holds.

Example 3. Let $\mathbf{N}=\{1,2,3, \ldots\}$ and $X=\mathbf{N} \cup\{\infty\}$. Let $\S$ be that topology on $X$ having base $\{\varnothing, X\} \cup\{\{1,2\},\{3,4\},\{5,6\}, \ldots\}$. By Zorn's Lemma expand $\varsigma$ to a topology $\mathcal{T}$ on $X$ maximal with respect to having no isolated points. Then $\bar{\top}$ satisfies all requirements of being nearly discrete except that of being $T_{0}$. To see that Lemma 1 fails for $\bar{Y}$, set $A=\{1,3,5, \ldots\}$ and $x=\infty$. Then $x \in A^{\prime}$, but $A \cup\{\infty\}$ is not a neighborhood of $x$ in $\tau$. Furthermore, it is easy to verify that Theorem 1 fails for $T$ by taking $\varphi=\mathscr{P}(X-\{\infty\})$, for then $\mathfrak{A}(\mathcal{G}, \mathcal{T})$ is not maximal.

In conclusion, we wish to indicate a purely ultrafilter-theoretic approach to maximal ideals which leads back to principal ideals modulo a set ideal. Note that $\mathcal{G}$ is a maximal ideal on $X$ iff $\mathscr{P}(X)-q$ is an ultrafilter, say $\mathscr{Q}(q)$, on $X$. One also has $q=\left\{A \mid X-A \in Q_{L}(q)\right\}$. In view of this an ideal $\mathfrak{A}(9, \widetilde{T})$, where 9 is a maximal ideal on $X$, can be written as

$$
\mathfrak{H}(q, \widetilde{T})=\{S \in \Sigma \mid\{x \in X \mid \mathfrak{N}(x, \mathcal{S}) \subseteq \mathfrak{N}(x, \mathcal{T})\} \in \mathcal{U}(\mathscr{S})\} .
$$

This motivates the following definition. Let $\mathscr{U}$ be an ultrafilter on $X$, and $\psi=\left\{\mathcal{U}_{x} \mid\right.$ $x \in X\}$ be a family of ultrafilters on $X$. Define

$$
\mathscr{H}(\mathcal{Q}, \psi)=\{S \in \Sigma \mid\{x \in X \mid \Re(x, \mathfrak{S}) \subseteq \mathcal{Q}\} \in \mathcal{Q}\} \text {. }
$$

Then $\mathfrak{B}(\mathcal{Q} L, \psi)$ is an ideal of $\Sigma$. We have proved the following facts about such ideals: (1) Every maximal ideal of $\Sigma$ which is principal modulo a set ideal may be written as $\mathfrak{B}(\mathcal{Q}, \psi)$ for some $\mathcal{Q}$ and $\psi$, and (2) every maximal ideal of $\Sigma$ having form $\mathfrak{B}(\mathcal{Q}, \psi)$ is principal modulo a set ideal. Since no new ideals of $\Sigma$ are obtained in this manner, we omit the proofs.

\section{REFERENCES}

1. O. Fröhlich, Das Halhordnungssystem der topologischen Räume auf einer Menge. Math. Ann. 156 (1964), 79-95.

2. E. Hewitt, A problem of set-theoretic topologv, Duke Math. J. 10 (1943). 309-333.

3. M. Katětov, On topological spaces containing no disjoint dense sets, Mat. Sb. 21 (1947), 3-12.

4. On nearly discrete spaces, Časopis Pěst. Mat. 75 (1950), 69-78.

5. A. R. Padmanabhan and B. V. Rao, Ideals in the lattice of topologies, J. Indian Math. Soc. 33 (1969), $65-71$.

6. . Corrections to the paper "Ideals in the lattice of topologies", J. Indian Math. Soc. 34 (1970). 287.

7. W. J. Thron and R. A. Valent, An embedding theorem for the lattice of $T_{1}$-topologies and some related cardinality results, J. London Math. Soc. (2) 9 (1975), 418-422.

Department of Mathematics, University of Colorado, National Center for Atmospheric RESEARCH, Boulder, COLORADO 80309 